\title{
Formation and Propagation of Temperature Anomalies along the North Atlantic Current**
}

\author{
Gerd Krahmann and Martin Visbeck \\ Lamont-Doherty Earth Observatory, Columbia University, Palisades, New York \\ GILLES REVERDIN \\ Laboratoire d'Etudes en Geophysique et Oceanographie Spatiales, Toulouse, France
}

(Manuscript received 7 February 2000, in final form 28 July 2000)

\begin{abstract}
A general circulation ocean model has been used to study the formation and propagation mechanisms of North Atlantic Oscillation (NAO)-generated temperature anomalies along the pathway of the North Atlantic Current (NAC). The NAO-like wind forcing generates temperature anomalies in the upper $440 \mathrm{~m}$ that propagate along the pathway of the NAC in general agreement with the observations. The analysis of individual components of the ocean heat budget reveals that the anomalies are primarily generated by the wind stress anomaly-induced oceanic heat transport divergence. After their generation they are advected with the mean current. Surface heat flux anomalies account for only one-third of the total temperature changes. Along the pathway of the NAC temperature anomalies of opposite signs are formed in the first and second halves of the pathway, a pattern called here the North Atlantic dipole (NAD). The response of the ocean depends fundamentally on $R_{t}=(L / v) / \tau$, the ratio between the time it takes for anomalies to propagate along the NAC $[(L / v) \sim 10$ years] compared to the forcing period $\tau$. The authors find that for NAO periods shorter than 4 years $\left(R_{t}>1\right)$ the response in the subpolar region is mainly determined by the local forcing. For NAO periods longer than 32 years $\left(R_{t}<1\right)$; however, the SST anomalies in the northeastern part of the NAD become controlled by ocean advection. In the subpolar region maximal amplitudes of the temperature response are found for intermediate (decadal) periods $\left(R_{t} \sim 1\right)$ where the propagation of temperature anomalies constructively interferes with the local forcing. A comparison of the NAO-generated propagating temperature anomalies with those found in observations will be discussed.
\end{abstract}

\section{Introduction}

Recent analyses of historical sea surface temperature (SST) data have revealed that coherent large-scale temperature anomalies occur in the North Atlantic Ocean on interannual to decadal timescales (Deser and Blackmon 1993; Kushnir 1994; Hansen and Bezdek 1996; Sutton and Allen 1997). A significant part of these anomalies are related to large-scale atmospheric forcing by the North Atlantic Oscillation (NAO) (Walker 1924; Walker and Bliss 1924), a seesaw of atmospheric mass between the polar and subtropical regions. The NAO is hence associated with changes in the large-scale atmospheric wind pattern, which in turn cause variations

\footnotetext{
* Lamont-Doherty Contribution Number 6099.
}

Corresponding author address: Dr. Gerd Krahmann, Lamont-Doherty Earth Observatory, Columbia University, Oceanography 206-C, RT 9W, Palisades, NY 10964-8000.

E-mail: krahmann@1deo.columbia.edu of the winter air and sea surface temperatures throughout the North Atlantic Ocean (Visbeck et al. 1998; Seager et al. 2000). Changes in the air-sea fluxes of heat and momentum during a positive NAO phase cool the subpolar and subtropical oceans while the temperatures off the North American east coast are warmer than normal.

The analysis of temperature anomalies at times when the NAO index is close to zero, that is, in quadrature to the NAO, indicates that the temperature anomalies found off the North American east coast propagate along the axis of the North Atlantic Current (NAC) (Visbeck et al. 1998). A similar movement of temperature anomalies originating from this region has been found in observations (Hansen and Bezdek 1996; Sutton and Allen 1997). The propagation speed derived from the observations is $1.7 \mathrm{~cm} \mathrm{~s}^{-1}$; this is significantly slower than the high velocities of the core of the NAC.

Sutton and Allen (1997) propose that the understanding of the processes that determine the propagation of the temperature anomalies along the NAC might be important for the prediction of interannual to decadal cli- 
mate variations over Europe. If the spreading of temperature anomalies is caused by advection in the ocean one might be able to skillfully predict the temperature evolution along the NAC. However, the effects of these temperature anomalies on the overlying atmosphere are still unclear (see Robinson 2000, for a recent review). It has been suggested that they can provide the missing memory that creates decadal variations of the NAO (Latif 1998).

In an analytical stochastic model Saravanan and McWilliams (1997) studied the response of a singlelayer constant-speed NAC to atmospheric forcing random in time but sinusoidal along the NAC. They found that the oceanic response falls into one of two regimes: a slow-shallow regime in which local effects are dominant and a fast-deep regime in which nonlocal advective effects are dominant. In the fast-deep regime the oceanic response peaks at a preferred frequency even though the original forcing spectrum was white. This frequency is determined by the NAC's advection speed divided by the length of the NAC. Saravanan and McWilliams (1997) find that for the observed advective velocity of the NAC of about $2 \mathrm{~cm} \mathrm{~s}^{-1}$ anomalies on the decadal timescale should be most prominent.

Here we use an ocean general circulation model (OGCM) coupled to an atmospheric mixed layer (Seager et al. 1995) in order to investigate the response of the North Atlantic Ocean to changes in the wind forcing. In contrast to models with prescribed surface heat fluxes the atmospheric mixed layer allows the model's surface heat fluxes to adjust to changes in atmospheric advection of temperature and humidity and in local SST anomalies. We have added NAO-like wind speed, vector, and wind stress anomaly patterns to climatological monthly forcing. The anomaly patterns have been modulated with idealized fixed frequency variations in order to study variation in response to different frequencies of the NAO. Some aspects of the overall oceanic response are discussed by Visbeck et al. (1998). Here we focus our attention on how these NAO-related temperature anomalies are formed and propagate along the NAC.

In subsequent sections we first describe the model and the different experiments performed and then analyze the model output and compare it to observational data.

\section{Numerical experiments}

\section{a. The model}

We have employed an ocean general circulation model that spans the Atlantic Ocean between $30^{\circ} \mathrm{S}$ and $73^{\circ} \mathrm{N}$ with a horizontal resolution of $2^{\circ}$ by $2^{\circ}$. The model has 30 fixed vertical levels, 13 of which are distributed over the upper $1000 \mathrm{~m}$ with decreasing vertical resolution from 24 to $100 \mathrm{~m}$. Below 2000-m depth, the vertical resolution is $250 \mathrm{~m}$ with a variable lowest layer thick- ness. The basin geometry and bathymetry are resolved on the model grid.

The model solves the primitive equations on an $\mathrm{A}$ grid and is forced by monthly mean wind stresses. Temperature and salinity are restored to climatological values over a few grid points near solid walls at the northern and southern ocean boundary. Freshwater fluxes are obtained by restoring the sea surface salinity to a monthly dataset with a 30-day relaxation timescale. The surface heat fluxes are determined by a prognostic atmospheric boundary layer model coupled to the ocean model's SST (Seager et al. 1995). The boundary layer atmospheric temperature and humidity are specified over land but vary over the ocean according to an advective-diffusive balance subject to air-sea fluxes. All other boundary conditions such as the shortwave radiation, cloud cover, wind speed, and wind vector are specified at each grid point with monthly resolution.

A simple 1/2-layer thermodynamic sea ice model is coupled between the ocean and atmospheric boundary layer in order to reduce the heat flux in ice-covered regions. Unresolved small-scale ocean processes are parameterized by a bulk wind-driven mixed layer model, convective adjustment, and isopycnal thickness diffusion.

First the model was initialized with climatological temperature and salinity data and integrated for 60 years with fixed climatological monthly forcing. The winter SST differences between the model and observations are typically less than $1^{\circ} \mathrm{C}$ with the exception of the Gulf Stream region. Its position is too far north and much of the cold slope water signal is missing in our simulations. Mixed layer depths are maximum during late winter in the Labrador Sea with a quite realistic distribution throughout the subpolar gyre.

In our experiments anomalies of wind stress and wind speed/vector have been added to the monthly forcing. In order to obtain a realistic circulation we used climatological mean forcing from Hellerman and Rosenstein (1983) for wind stress and from the National Centers for Environmental Prediction-National Center for Atmospheric Research (NCEP-NCAR) reanalysis for wind vector and speed, respectively. The anomalies added to the climatological part of the forcing are an idealized form of the wind variations associated with the NAO. The anomaly fields were derived from the NCEPNCAR reanalysis (1958-98) data by regressing the winter NAO index of Hurrell (1995) against the November through April wind speed, wind vector, and wind stress fields. The wind vector and wind stress anomaly fields exhibit enhanced westerlies during the positive NAO phase accompanied by a weakening of the trade winds (see Fig. 1a). The wind speed anomalies show a roughly zonally banded structure with significant large-scale variability throughout the subtropical and subpolar Atlantic (see Fig. 1c). The NAO-related anomalies explain a significant fraction of the total variances. For the zonal wind stress component (Fig. 1b) we find that more than 

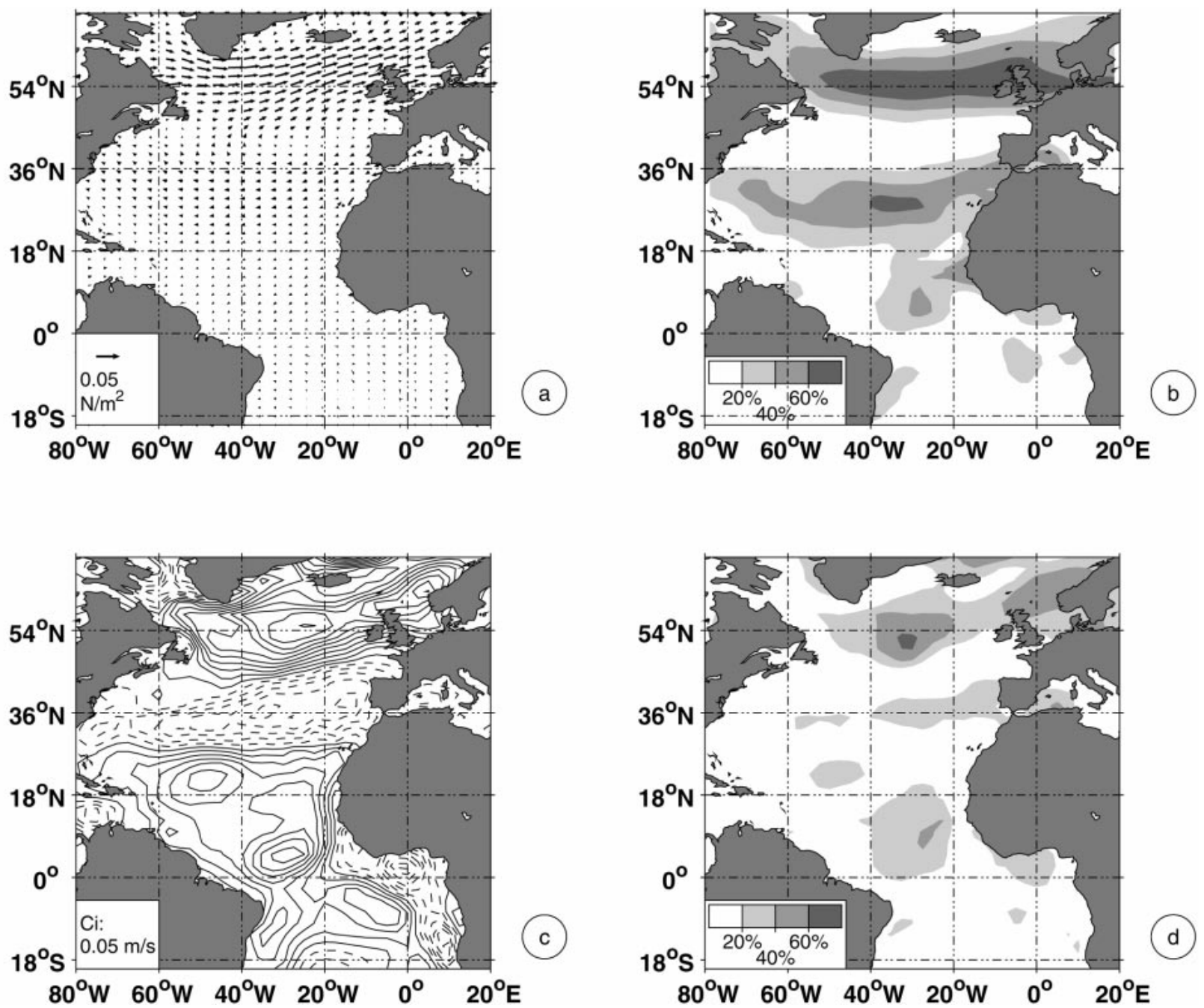

FIG. 1. Wind stress (a) and wind speed (c) forcing anomaly fields used for the experiments. The anomaly fields have been derived by regressing the wind stress and wind speed of the NCEP-NCAR reanalysis onto the NAO time series. The two displays on the right show the fraction of the total variance explained by the NAO-regressed anomalies for the zonal component of the wind stress (b) and the wind speed (d), respectively. For all calculations winter averages (Nov-Apr) have been used.

$60 \%$ of the total variance is explained in subpolar and subtropical latitudes while for the wind speed (Fig. 1d) the numbers are somewhat lower.

The idealized NAO-like wind anomalies were modulated with a sine wave of 2-64 yr period, which allows us to isolate interannual, decadal, and multidecadal variations of the NAO (denoted NAO-D in Table 1). Note that, as a compromise between realistic and idealistic scenario, the anomalies were only applied during the winter season (Nov-Apr), when the NAO is responsible for most of the sea level pressure variance (e.g., van Loon and Rogers 1978). Each experiment was run over several forcing cycles so as to obtain a quasi-equilibrium upper-ocean model response.

For a second set of experiments (NAO-M) we suppressed all explicit oceanic advection in order to ex-

TABLE 1. List of experiments conducted with the Lamont Ocean Atmospheric Mixed Layer Model.

\begin{tabular}{llcc}
\hline \hline \multicolumn{1}{c}{ Run } & Model & Forcing modulation & Anomaly fields \\
\hline NAO-D2 to NAO-D64 & Dynamical & $2-64$ yr sinus & NCEP NAO-regr. \\
NAO-M2 to NAO-M64 & Mixed layer & $2-64$ yr sinus & NCEP NAO-regr. \\
TRACER & Dynamical & No anomaly fields added \\
NAO-HURR & Dynamical & NCEP NAO-regr. \\
NCEP & Dynamical & NCEP anomaly fields \\
NCEP-M & Mixed layer & NCEP anomaly fields \\
\hline
\end{tabular}


amine a mixed layer model's response to surface fluxes only. The mean ocean heat flux divergence was prescribed as a monthly flux with no interannual variability.

We also performed a run without adding forcing anomalies (TRACER) in which a tracer patch was released in the formation area of the temperature anomalies to study the pure advective spreading.

Finally, we analyzed three more realistic experiments: One used the observed time series of the NAO to modulate the NAO-like wind anomalies (NAO-HURR); second, we applied the full wind and wind stress anomalies of the NCEP-NCAR reanalysis (NCEP); and a third experiment where we suppressed the oceanic advection while we added the full wind and wind stress anomalies of the NCEP-NCAR reanalysis (NCEP-M). Some results of the latter two integrations are reported by (Seager et al. 2000).

\section{b. Simulation of NAO-induced SST anomalies}

Visbeck et al. (1998) have shown that our ocean model is able to generate the observed SST tripole response when forced with NAO-like wind anomalies. They also report that the anomaly fields are not stationary, they propagate along the path of the NAC. Figure 2 shows the evolution (half a cycle of the forcing) of SST anomalies for a 12-yr NAO forcing period. One can clearly see the propagating SST anomalies.

Lagged correlations of observed SSTs showed that temperature anomalies formed off the North American east coast follow approximately the pathway of the NAC across the North Atlantic (Sutton and Allen 1997). We performed a similar analysis of the NAO-D12 run and show the lagged correlations in Fig. 3. The result is qualitatively similar to that of the observational study of Sutton and Allen (1997). To investigate whether the reference area of Sutton and Allen (1997) is perhaps not the source area of the anomalies we extended the correlation to preceding lags but found them not to be significant (not shown). This means that the observed temperature anomalies found in the NAC are created in or around the reference area or farther downstream in the NAC. Our model experiments similarly show no clear sign of anomalies upstream the reference area.

For the subsequent analyses we will inspect the response along the model's NAC (see Fig. 3 for the location of the pathway). We found that the Hovmöller diagrams of observational data show comparable temperature anomalies for both the model's NAC and the one originally used by Sutton and Allen (1997). Thus we employ the same pathway for both observational and modeled data.

\section{Results}

In this paper we seek to answer two main questions:

- How are the temperature anomalies generated that seem to propagate along the path of the North Atlantic Current?-air-sea fluxes versus changes in upperocean heat transport divergence

- What causes the apparent propagation?-oceanic advection versus phase-lagged local forcing.

We will address these questions in the framework of a series of numerical experiments with emphasis on

- how the model's response depends on the frequency of the idealized forcing

- How the model's response to the idealized NAO-like forcing compares with more realistic model runs and with observations.

We begin with a discussion of run NAO-D12 where the NAO-like wind anomaly was added to the full ocean model with a fixed frequency of $12 \mathrm{yr}$. We first examine the different components contributing to the heat budget along the pathway of the NAC. This will shed some light on the generation and propagation of temperature anomalies. The 12-yr forcing period was chosen because we found that the model SST response was not unlike that observed (Visbeck et al. 1998). We will show that the conclusions drawn from the 12 -yr period are, however, with adjustments for amplitude and timing, also valid for the other forcing periods.

\section{a. Formation and propagation of temperature anomalies}

In the following we will make extensive use of timespace (Hovmöller) diagrams to investigate the evolution of temperatures, their time derivatives, and some of the components contributing to temperature changes. In all of these diagrams the mean annual cycle has been removed and the interannual monthly anomalies are shown. For example Fig. 4 shows different properties along the NAC pathway for one complete 12-yr forcing cycle. The wind anomaly associated with positive and negative NAO anomalies has reached its maximum in the winters of years 3 and 9, respectively, and is zero in years 0 and 6 . We show both the temperature anomalies of the uppermost model layer (0-24 m, the model's SST) and the heat content of the upper $440 \mathrm{~m}$ (HC), which is proportional to the upper-ocean average temperature. Most of the model's response to interannual changes in wind and heat flux is contained in the upper $500 \mathrm{~m}$ and is thus well represented by the $\mathrm{HC}$ fields.

A casual inspection of the SST and HC fields (Fig. 2 and Figs. 4a and 4b) gives a clear indication for propagation of temperature anomalies along the NAC. In Figs. $4 \mathrm{a}$ and $4 \mathrm{~b}$ they are visible as a broad band of positive anomalies extending diagonally across the figure. Such a pattern is very similar to the ones derived from observed SST anomalies as shown by Hansen and Bezdek (1996); Sutton and Allen (1997). It is worth noting that in the western part of the region the warm anomalies are in phase with the forcing (relative to a 

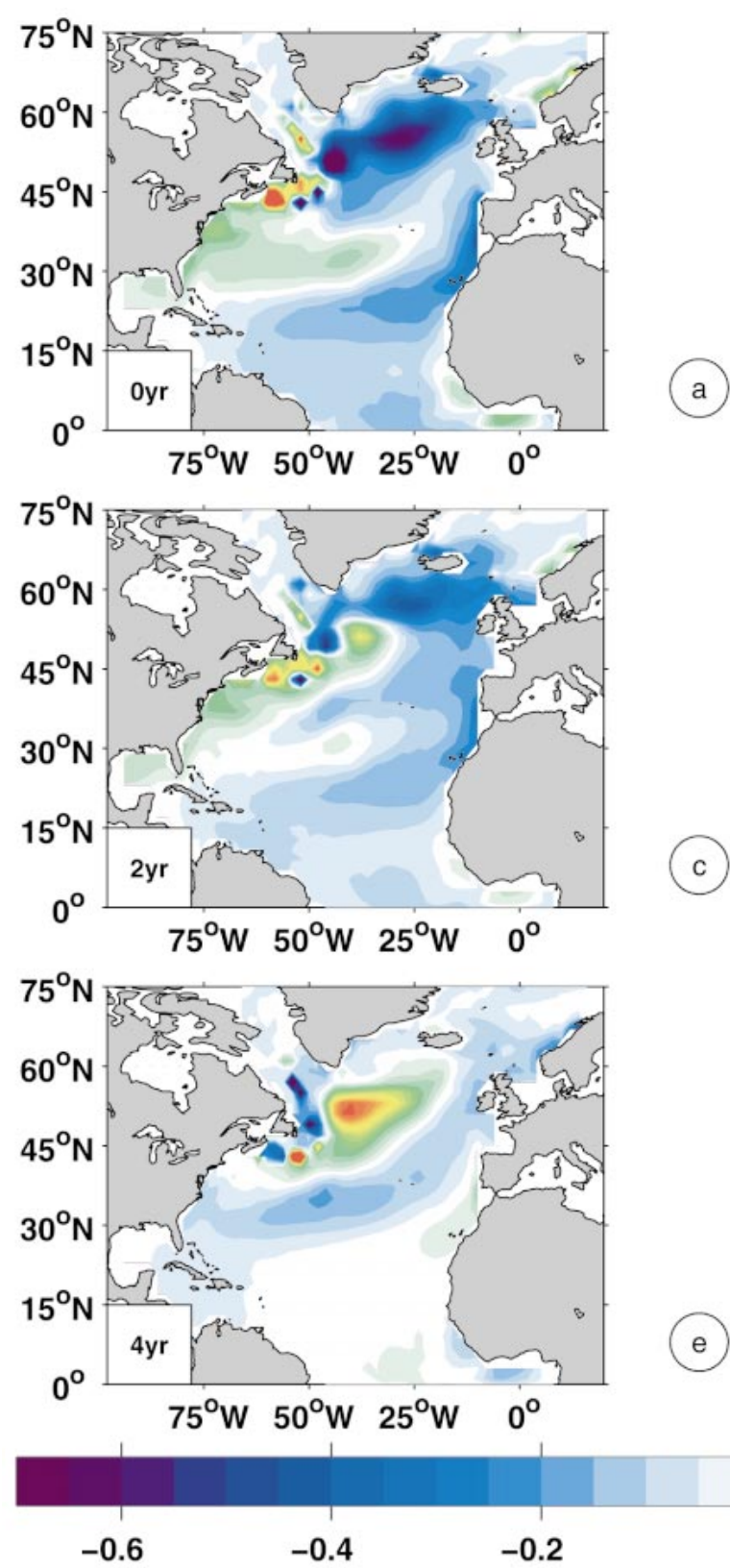
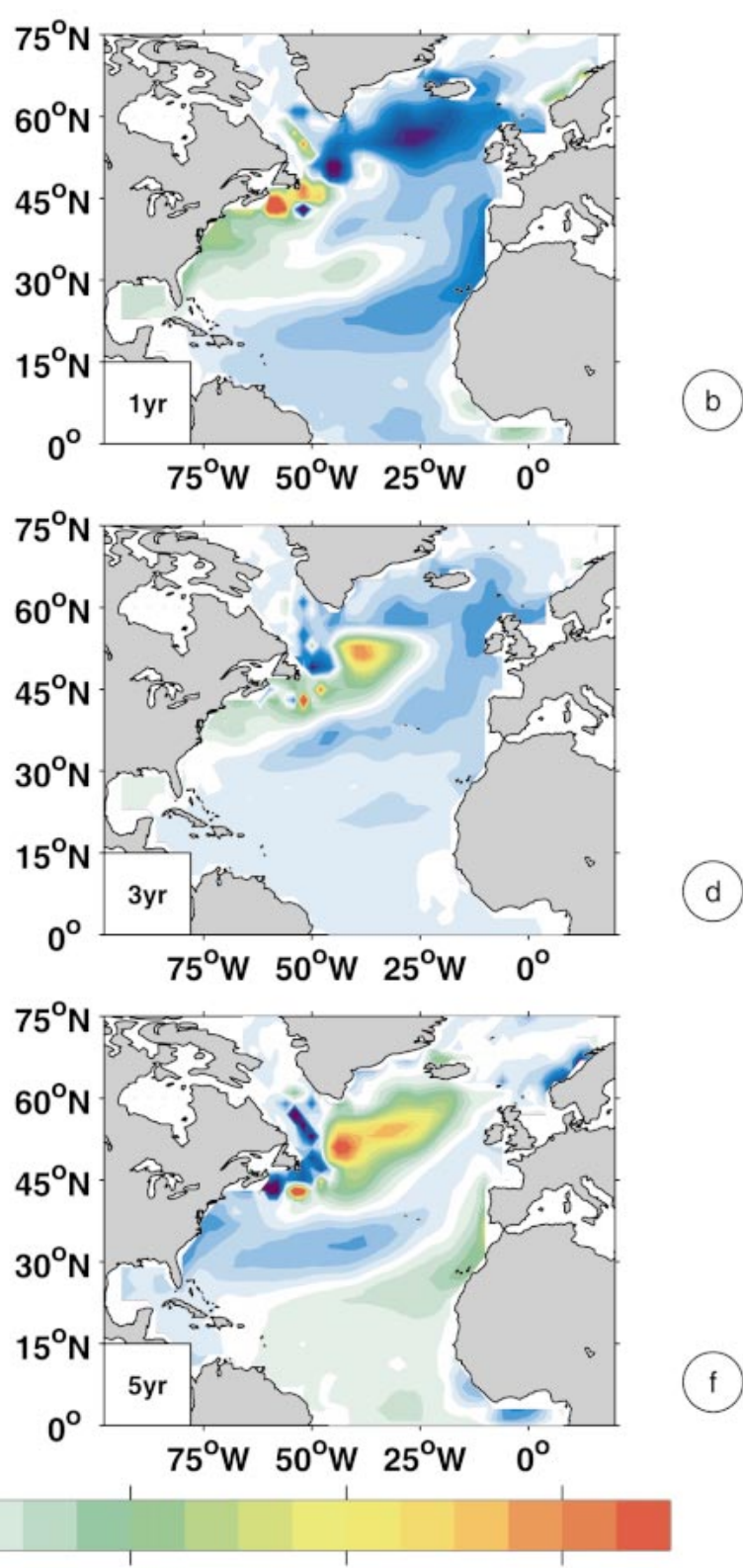

0.4

0.6

JFM SST anomaly $\left[{ }^{\circ} \mathrm{C}\right]$

FIG. 2. Sea surface temperatures of the experiment NAO-D12 zero, one, two, three, four, and five years after the sinusoidal forcing maximum $(\mathrm{NAO}+)$. In response to the idealized wind and wind stress anomalies a temperature anomaly is formed off the North American east coast. This anomaly apparently propagates along the pathway of the NAC.

positive NAO index) and in the eastern part, about 6500 $\mathrm{km}$ downstream, of opposite sign. Hence we sample with the NAC pathway the upper two lobes of the NAO SST tripole, which we call the North Atlantic dipole (NAD).

The temperature anomalies in Figs. $4 \mathrm{a}$ and $4 \mathrm{~b}$ cannot be explained by only a direct response to the wind anomalies. We find that the tilt of the anomaly band is roughly constant over the whole NAC pathway, indicative of a constant propagation speed of a few centimeters per second. When the positive anomaly has arrived at the eastern end of the pathway, after about 4-6 years, a negative anomaly emerges at its beginning. From this we can draw two immediate conclusions: 1) The continuous existence of the temperature anomalies means that they can persist during years with weak or no wind anomalies (i.e., years 0 and 6 for the 12 -yr NAO period). In other words the ocean exhibits a significant amount 


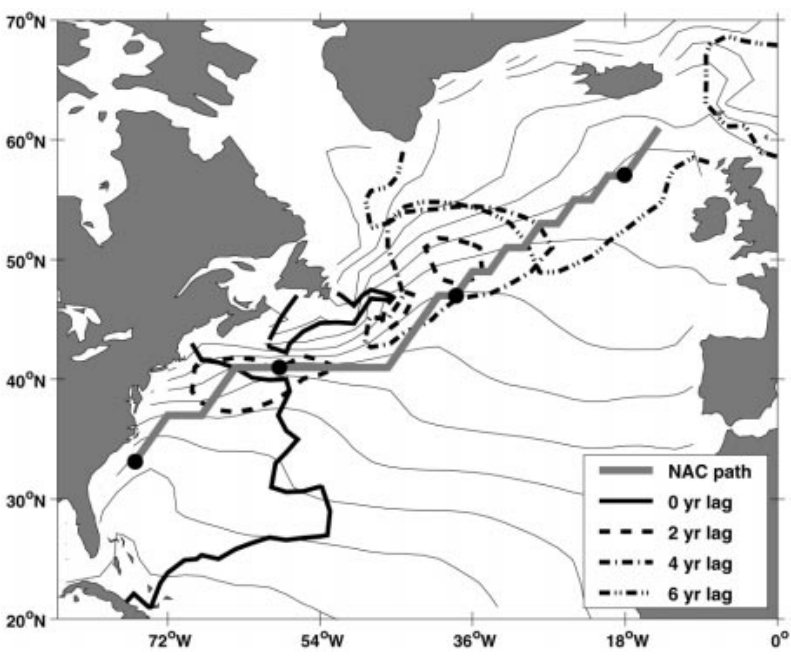

FIG. 3. Lagged correlations of monthly SST anomalies for the 12yr idealized NAO period. The lagged correlations are calculated similarly to those of Sutton and Allen (1997), using the SSTs in the area $31^{\circ}-39^{\circ} \mathrm{N}, 60^{\circ}-80^{\circ} \mathrm{W}$ as reference. We did, however, not apply a $3-\mathrm{yr}$ running mean filter to our modeled data. Contoured is a correlation of 0.75 . Also shown is the NAC's pathway as used in our analyses. The filled circles denote $0,2000,4000$, and $6000 \mathrm{~km}$ distance from the beginning of the pathway.

of memory lasting over a few years. 2) Since the inclination of the band is about constant even during times of weak forcing, oceanic advection of existing temperature anomalies by the mean currents must play a fundamental role in the apparent propagation.

In addition to the broad band of positive SST anomalies visible in Fig. 4a the Hovmöller diagram also exhibits distinct horizontal stripes, which seem to be modulated on seasonal timescales. These stripes occur most pronounced during the forcing (winter) season. Two mechanisms could be responsible: anomalous forcing or deepening of the oceanic mixed layer whereby deep temperature anomalies reemerge at the sea surface. Since we find the seasonal variations throughout the experiment and not only during the years of maximum forcing (years 3 and 9), the deepening of the mixed layers during the winter season must be an important process in generating the seasonal SST anomalies. This implies that the upper ocean heat content is the more appropriate parameter to analyze when studying the propagation of temperature anomalies.

The heat content anomalies similarly show seasonal variations in the form of stripes. In contrast to the stripes of the SST pattern they are inclined, indicating that these seasonal anomalies are propagating. Interestingly the propagation speed of the seasonal signals differs from those of the broad interannual band. This suggests that different processes are responsible for the apparent propagation on seasonal and interannual timescales. Hovmöller diagrams of the heat content changes for other forcing periods (not shown) reveal that the inclination of the seasonal variations is constant, while the incli- nation of the interannual anomalies varies with the forcing period. Thus we propose that the mean oceanic advection, which we have found to be largely independent of the forcing period, is responsible for the propagation of the seasonally varying anomalies.

To test this hypothesis we have integrated the model's upper-ocean velocities along the NAC pathway and show the resulting time-distance relation as an additional black line in Fig. 4h. This idealized advection path agrees reasonably well with the slope of the seasonal HC anomaly stripes.

Let us now examine which terms of the oceanic heat transport divergence contribute to the heat content changes along the NAC pathway. Figures $4 \mathrm{c}-\mathrm{f}$ show different contributions to the heat content tendency due to the advection of the mean temperature by anomalous currents $u^{\prime} \nabla T$ and due to the advection of anomalous temperatures by the mean currents $u \nabla T^{\prime}$ both for SST (left column of graphs) and $\mathrm{HC}$ (right column). The heat content tendencies of the two layers (i.e., the time derivatives of the heat contents in Figs. $4 \mathrm{a}$ and $4 \mathrm{~b}$ ) are displayed for reference in Figs. $4 \mathrm{~g}$ and $4 \mathrm{~h}$, respectively. Furthermore, the surface heat flux anomalies are shown in Fig. 4i. The sum of the two advection terms plus the air-sea heat flux can explain most of heat content changes with only a small contribution from the mixing terms. Note that $u \nabla T^{\prime}$ and $u^{\prime} \nabla T$ have been derived from the monthly averaged data since we had no easy way to assess the contribution of short-term variations of temperature and currents. However, one can show that these terms cannot be very large in our coarse resolution model.

Let us first inspect the surface heat fluxes (Fig. 4i) in comparison to the SST and $\mathrm{HC}$ tendencies (Figs. $4 \mathrm{~g}$ and $4 \mathrm{~h}$ ). If we compare Figs. $4 \mathrm{i}$ and $4 \mathrm{~h}$, we find that the amplitudes of the surface heat fluxes account for only one-third of the heat content changes of the upper $440 \mathrm{~m}$. Thus for the deeper layers the advective terms are a large source of temperature anomaly and, in fact, are more important than the surface heat fluxes. For the surface layer (Fig. 4g) we find that the heat flux and the total change are of the same order of magnitude but occur at different times, again implying that the oceanic contribution is important. Please note, that in order to get an estimate of the heat content changes of the surface mixed layer we multiplied the heat content of the 24-mthick uppermost model layer by three.

We now continue with the analysis of the $u \nabla T^{\prime}$ and $u^{\prime} \nabla T$ patterns in Fig. 4. One finds that only the component with the advection of anomalous temperatures by the mean flow $\left(u \nabla T^{\prime}\right)$ exhibits inclined isotherms on the seasonal timescale, which are the sign of oceanic advection. In contrast $u^{\prime} \boldsymbol{\nabla} T$ has mostly nonpropagating isotherms, which points to its important role during the winter forcing season. In the surface layer the variations of the component $u^{\prime} \nabla T$ follow roughly the sinusoidal NAO forcing while in the layer $0-440 \mathrm{~m}$ the modulation is not quite as clear. 

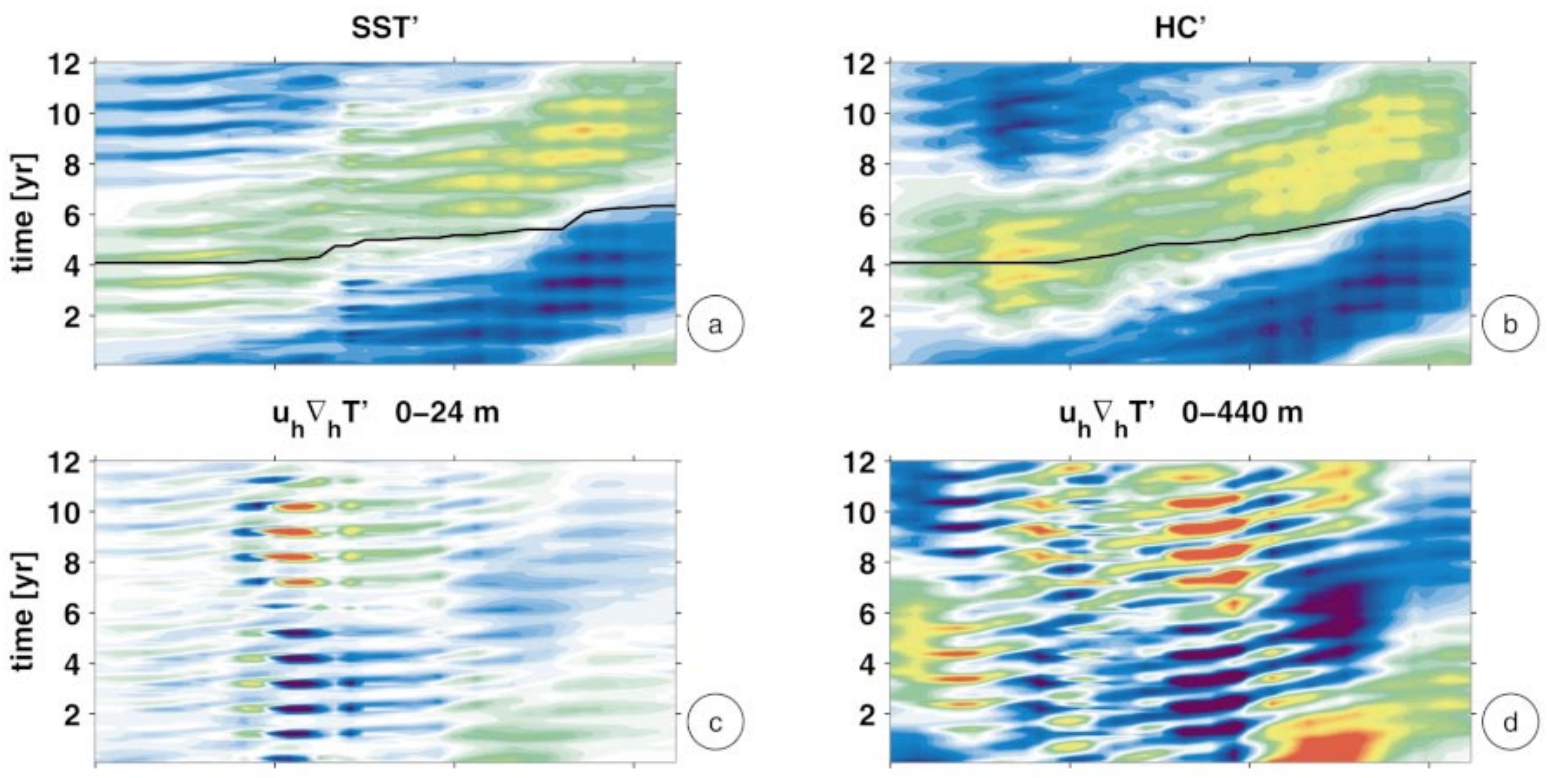

$$
u_{h}{ }^{\prime} \nabla_{h}{ }^{\top} \quad 0-24 m
$$
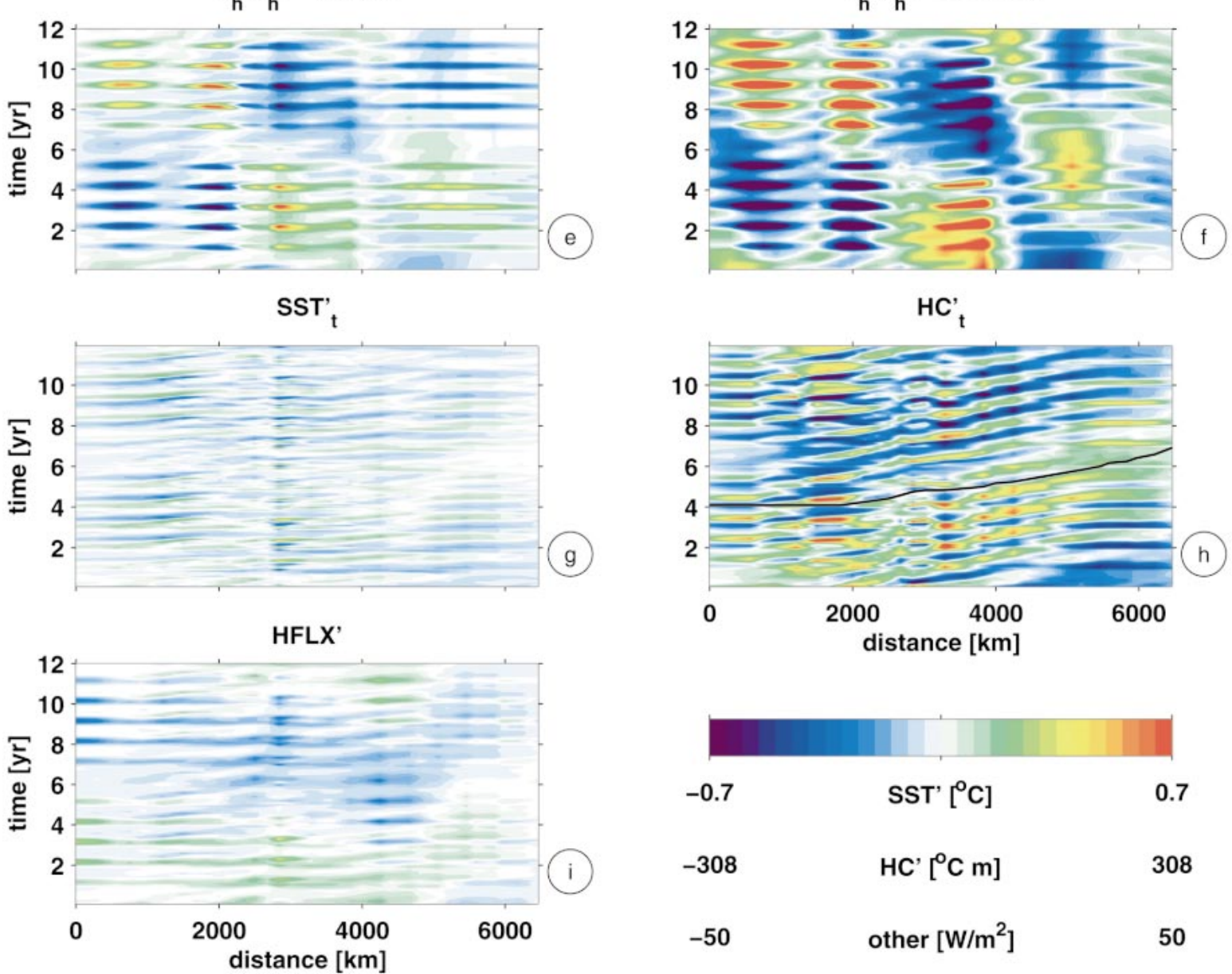

FIG. 4. Sea surface temperature and upper 440-m heat content anomalies of the experiment NAO-D12 along the pathway of the NAC (graphs a and $b$ ), their tendency (graphs $g$ and $h$ ), and components contributing to their tendency. Shown is always one full cycle of the forcing. To make the SST tendencies in the graphs $\mathrm{c}$, e, and g comparable to the heat content change and the surface heat flux it has been assumed that the temperature change in the uppermost model layer is representative of the mixed layer, here assumed to be three times as thick as the uppermost layer (i.e., 72 $\mathrm{m})$. The black lines in graphs $\mathrm{a}, \mathrm{b}$, and $\mathrm{h}$ show how a tracer patch inserted at the beginning of the pathway propagates along the pathway. 
An important characteristic of the component $u^{\prime} \nabla T$ within the surface layer is its $180^{\circ}$ phase jump in the response between the first half and the remaining part of the NAC pathway. This characteristic forms, together with the advection along the pathway, an environment much like the one proposed by Saravanan and McWilliams (1997). This aspect is most pronounced in SST but still visible in the HC pattern, especially during the wintertime forcing. In the $\mathrm{HC}$ pattern in the last third of the NAC pathway we also find significant anomalies during times of weak NAO forcing (both during the summer season and during zero crossings of the sinusoidal modulation). These are the signs of geostrophic currents resulting from temperature anomalies. They do not follow the dipolar pattern and thus complicate their interpretation.

At some places along the pathway (e.g., 3500 and $5000 \mathrm{~km}$ ) the contributions from the components $u^{\prime} \nabla T$ and $u \nabla T^{\prime}$ are opposing each other. This behavior can be interpreted as anomalous temperatures being created by the wind stress forcing (i.e., $u^{\prime}$ ) and then being advected along the NAC by the mean current $u$.

In summary, we find that the temperature anomalies at the beginning of the NAC pathway are generated by a mixture of atmospheric heat flux and momentum forcing. In particular the deep reaching temperature signals are to a large extent generated by changes in the ocean currents that alter the local heat transport divergence. Once generated, parts of the signal are advected with the mean flow northeastward with a speed of a few centimeters per second. However, there was a clear difference in the propagation speed of the seasonal and interannual signals. While we could readily understand the advection of the seasonal signal as a direct consequence of the mean currents, the interannual propagation deserves closer inspection. In order to accomplish this we have investigated the response of the ocean model as a function of the forcing frequency.

\section{b. Variable forcing periods}

The analysis of observational data has shown that SST anomalies propagate along the NAC (Sutton and Allen 1997) on decadal timescales. The NAO forcing, however, exhibits significant variability over a range of frequencies ranging from interannual to multidecadal. Thus we decided to perform a whole series of ocean response experiments with forcing periods ranging from 2 to 64 years (see Table 1). Visbeck et al. (1998) showed that the amplitudes of the ocean model's SST response depends on the period of the forcing. In particular, parts of the subpolar gyre showed a strongly enhanced response at periods between 8 and 24 years. The subtropical Atlantic and Gulf Stream regions, however, showed increasing energy for longer periods, a slightly red response spectrum.

Figure 5 displays the upper heat content, surface heat fluxes, and oceanic heat transport divergence (defined as the difference between the air-sea heat flux and the time derivative of the upper 440-m ocean heat content) along the pathway of the NAC for selected NAO periods from 4 to 64 years. Shown is always one full idealized NAO cycle with a zero wind anomaly at year 0 . It is obvious that the ocean's response changes significantly with the period of the forcing. For the periods between 8 and 32 years we find broad inclined bands of interannual temperature anomalies similar to that of the 12yr period described in the previous section. The 4-yr period, however, exhibits a more checkerboard-like pattern while the 64-yr period suggests a more in-phase response. Similar changes are found in the surface heat fluxes and implied oceanic flux fields (Fig. 5, middle and right columns).

\section{1) SEAsonal Forcing AND DeCAy CyCle}

The surface heat fluxes for the 4-yr period (Fig. 5b) reveal two distinct regimes. During times with active forcing (Nov-Apr, indicated by the black graph at the left side of the panel) the first half of the pathway $(0-3000 \mathrm{~km})$ experiences positive surface heat fluxes while the second half (4000-6500 km) shows negative heat fluxes for a positive NAO index. The pattern corresponds to the southwest-northeast dipole that connects the western subtropical gyre with the eastern part of the subpolar North Atlantic (Fig. 3). During the summer season when there is no anomalous forcing (MayOct) the surface heat fluxes still show the NAD pattern. However, the heat fluxes are of opposite sign compared to the forcing season. One can easily see that during the summer the surface heat fluxes are damping the heat content anomalies created in the preceding winter season. Anomalous warm temperatures thus lead to enhanced surface heat loss, they provide a negative feedback.

Next we inspect the contribution of ocean dynamics. As shown in the previous section, anomalous momentum input from changes in the wind stress fields can cause significant changes in the oceanic heat transport and its divergence. Much of this effect is due to changes in the Ekman transport but there are also contributions from the geostrophically adjusting deeper flow. Those mechanisms as well as changes in the vertical and horizontal mixing are included in the implied fields shown in the right column of Fig. 5. This contribution to the upper-ocean heat content changes similarly exhibits two regimes. During winter (active forcing season) a pattern emerges that is quite similar to that of the surface heat fluxes: positive heat content changes in the first half of the pathway and negative in the second half. Thus for short NAO forcing periods the surface heat fluxes reinforce the oceanic contribution, resulting in a strong temperature signal. During summer, however, the oceanic contribution differs from the surface heat fluxes: we find negative contributions preceding negative heat content anomalies and positive contributions following 

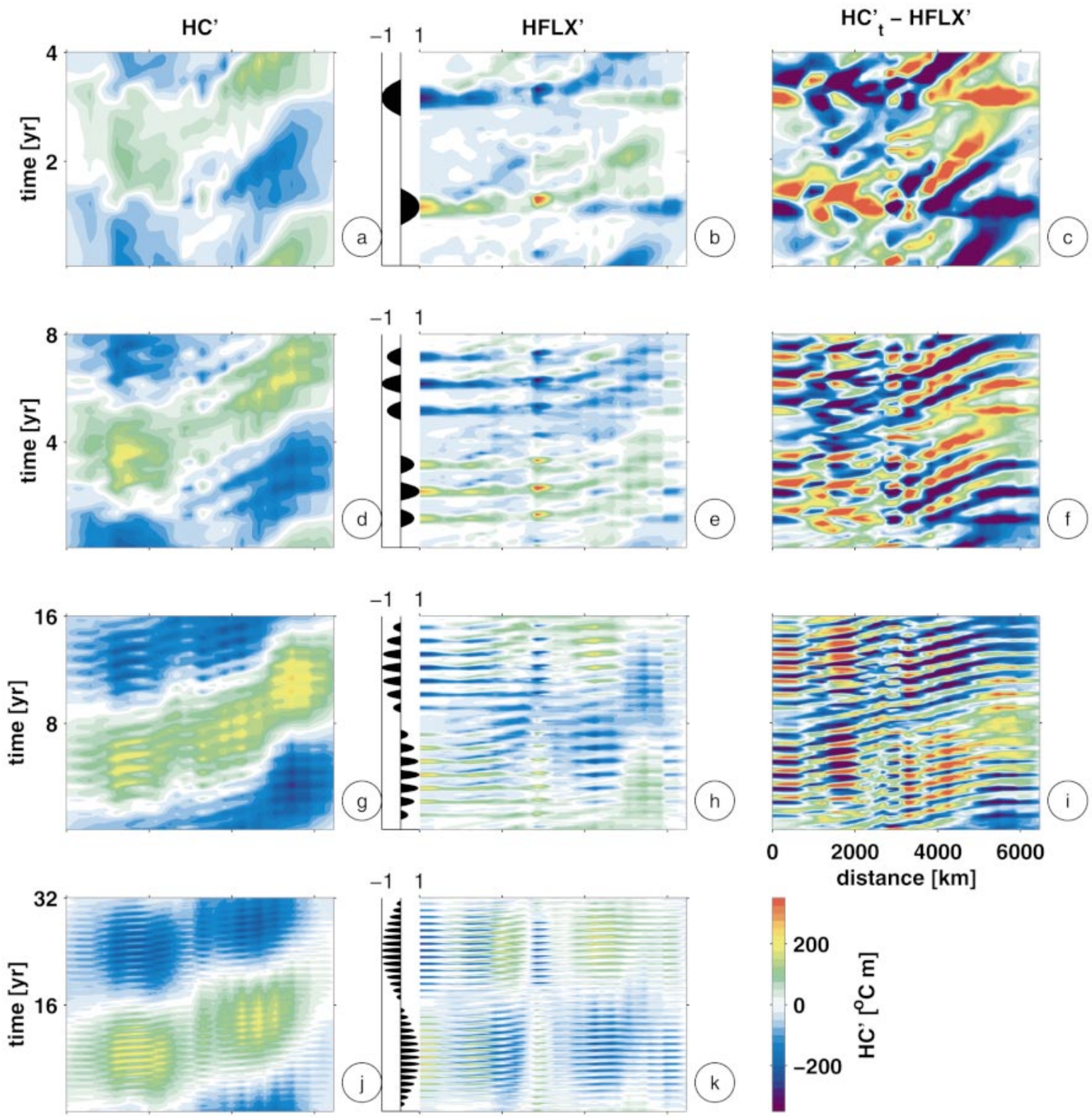

$-1 \quad 1$
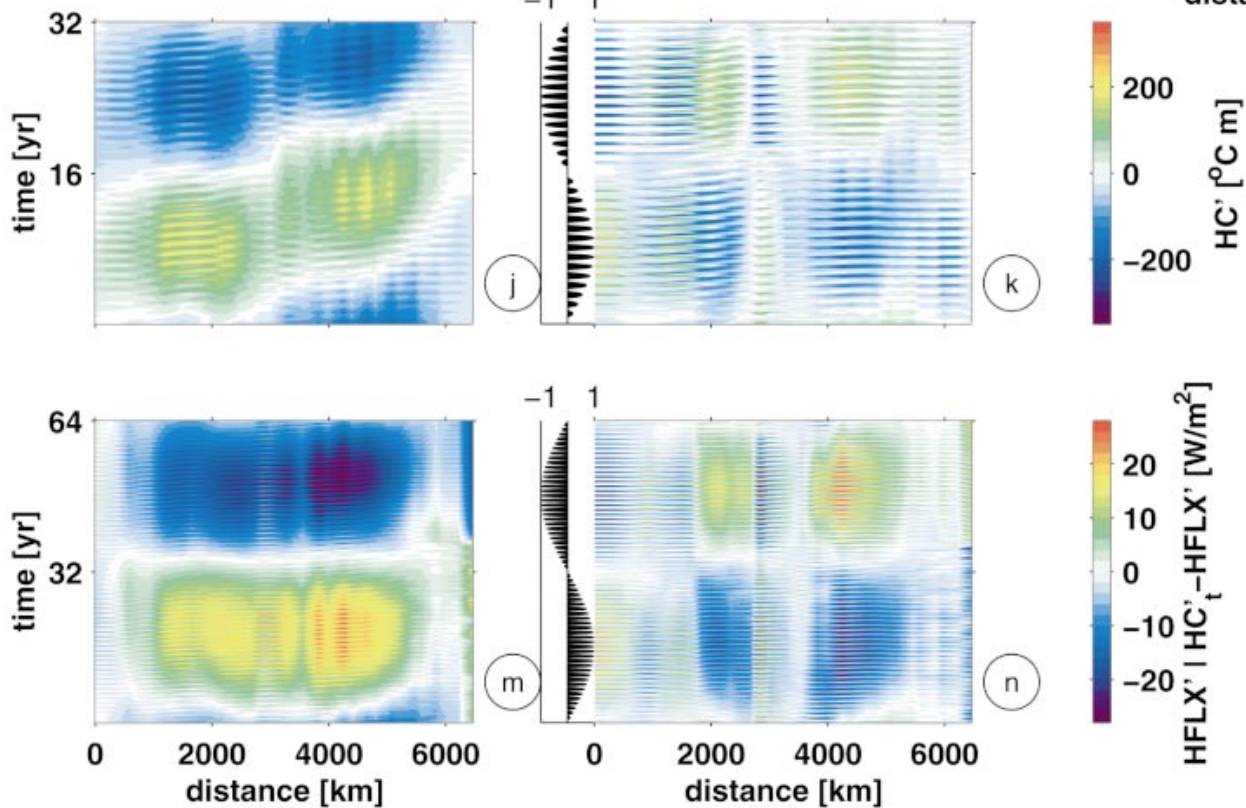

FIG. 5. Upper-ocean heat content, surface heat flux, and oceanic heat transport divergence for selected NAO periods. Shown is again one full cycle of the sinusoidal forcing. The forcing modulation is displayed between first and second column. The heat transport divergence has been determined by subtracting the surface heat fluxes from the total heat content changes. 
them and similar patterns for the opposite signs while the heat fluxes are always opposing the temperature anomalies. This suggests that the heat content anomalies are mainly formed by the oceanic heat transport divergence and not the surface heat fluxes. The oceanic contributions exhibit, just like the heat content, inclined isotherms during periods without active forcing. As we have shown in the previous section these inclined isotherms arise from oceanic advection relocating temperature anomalies that were formed during the winter period either by changes in ocean heat transport divergence or to a smaller extent by surface heat fluxes.

We conclude that during the summer ocean dynamics advect heat content anomalies downstream toward the northeast along the path of the NAC, while surface heat fluxes are damping the anomalies.

\section{2) INTERANNUAL SIGNALS}

In the following we compare the response of the model to different forcing periods shown in Fig. 5. The Hovmöller diagrams for the 4-yr and 8-yr NAO periods show the heat content anomalies arranged in a checkerboard-like pattern. For the 8-yr period a diagonal connection between the two lobes of the NAD is more clearly visible than for the 4 -yr period. The heat flux patterns for the 8-yr period are largely similar to those of the 4-yr period with the exception of a mostly damped response in the northern part of the pathway. This means that the surface heat fluxes in this part of the pathway change from a partially active role for the 4-yr NAO period to a mostly passive role for the $8-y$ period. The oceanic contribution to the heat content changes is largely similar for both shorter NAO periods with a pronounced NAD pattern during times of active forcing (winter). In the central part of the pathway we can observe the gradual change from predominantly local forcing to advectively determined temperature anomalies. While for the 4-yr NAO period there is barely any diagonal connection between the two lobes of the NAD, the 8-yr period shows some signs of temperature anomalies being advected from the first half of the pathway into the second. This is even more pronounced for the 16-yr NAO period in Fig. 5i. The finding that, in the right column of Fig. 5 with increasing forcing period, continuous inclined isotherms start to dominate the oceanic contribution means that temperature anomalies formed in the first half of the pathway and advected downstream outgrow the anomalies that are created locally in the second half.

For longer forcing periods (16 and 32 years) we find that the anomalies of the southwestern part of the NAD remain in phase with the forcing, while the anomalies of the northeastern part occur earlier with respect to the NAO forcing phase. In the 64-yr experiment the response of the subpolar part has shifted so much that it appears to be in phase with the external forcing. Hence we loose the signature of the NAD.
How can we interpret the patterns found in Fig. 5? In our experiments two basically different processes generate heat content anomalies along the pathway of the NAC: the local formation of temperature anomalies both by surface heat fluxes and heat transport divergences, and the advection of existing temperature anomalies along the pathway. These two processes have a different temporal scaling. The first follows the externally prescribed period of the NAO-like forcing, while the second is determined by the model's advection. We can define a nondimensional number that compares the two timescales:

$$
R_{t}=\frac{(L / v)}{\tau}
$$

where $L$ is the length of the pathway, $\tau$ represents the forcing period, and $v$ is the propagation speed of the temperature anomalies. The propagation speed to be used in this calculation is the effective speed with which the temperature anomalies propagate along the NAC. Because of diffusion and damping by the overlying atmosphere this speed is significantly slower than the surface currents of the NAC. Here we use a speed of $2 \mathrm{~cm}$ $\mathrm{s}^{-1}$ as found in observations, which is about half the average advective speed of the 0-440 m layer or onethird of the surface currents in our model.

For the case that the two timescales agree we expect constructive interference while for disagreeing timescales destructive interference might be expected. However, since the temperature anomalies created by the two processes are not necessarily of the same magnitude, a total cancellation of the temperature anomalies in the second half of the pathway, where both processes play a role, is rather unlikely. Indeed we are able to support the outlined scenario. For the $4-y r$ period it is rather unclear whether we have constructive or destructive interference. But for the 8-yr period we have clear support for constructive interference. For this NAO forcing period we can identify both the heat content anomalies caused by local formation as checkerboard pattern and the advection of existing temperature anomalies as diagonal stripes. The diagonal stripes connect the anomalies of the checkerboard pattern, indicating that the timescales of the two processes agree for this forcing period. If we continue to look at longer timescales, we find for the 16-yr period that diagonal stripes (i.e., advected temperature anomalies) again extend downstream from the temperature anomalies formed in the first half of the pathway. For this forcing period the timescales of advection and external forcing do not match perfectly. This results in a step in Fig. $5 \mathrm{~g}$ between the broad advective diagonal temperature anomaly band and a remaining patch resulting from the local forcing in the last quarter of the NAC's pathway. The two timescales do, however, not disagree enough to cause significant destructive interference, which can first be seen for the 32-yr forcing period. There only the broad di- 

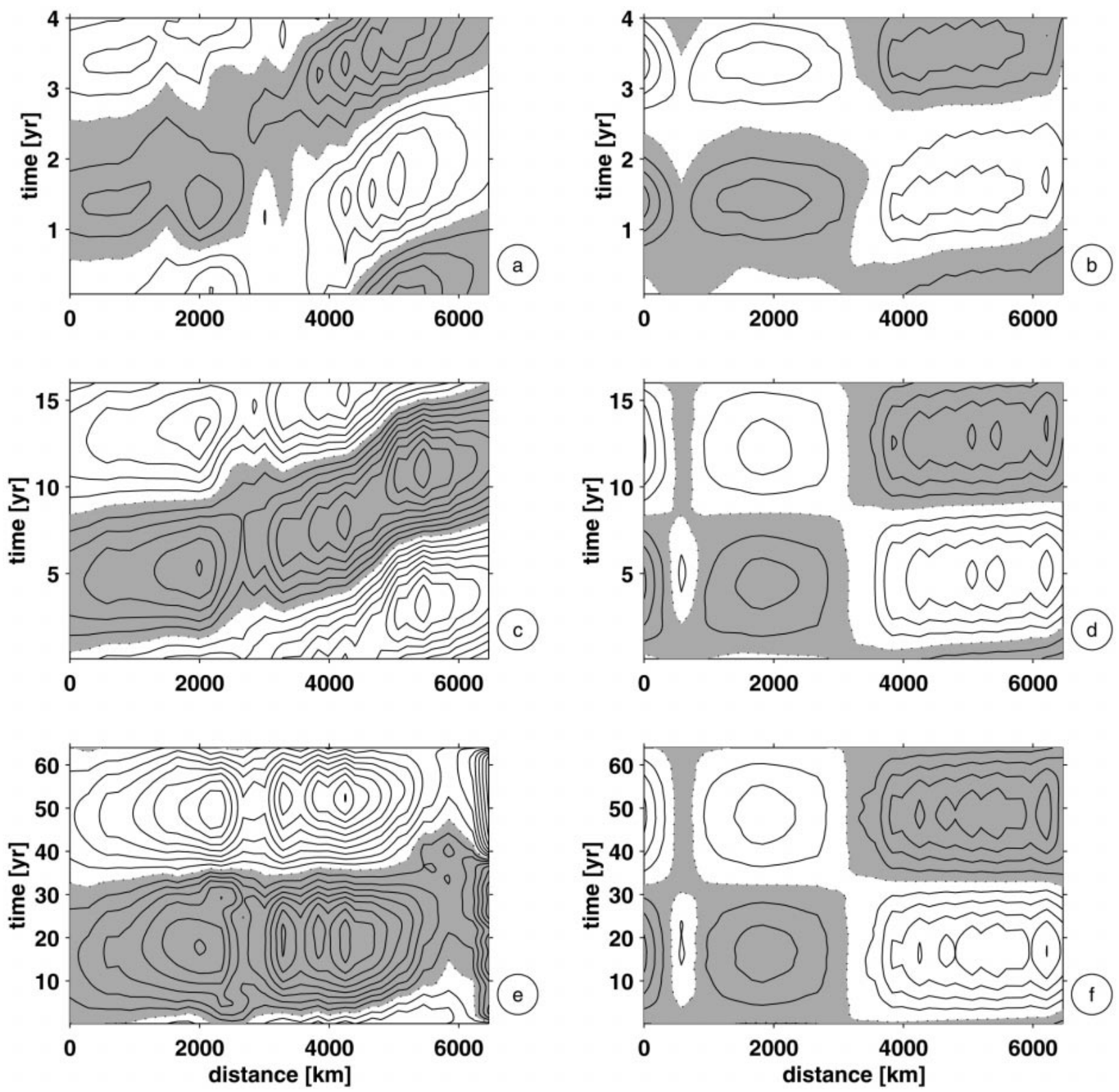

FIG. 6. Heat content anomalies along the NACs pathway for the advective (left column, NAO-D) and nonadvective (right column, NAO-M) ocean. Shown are forcing periods of 4,16 , and 64 years. The data has been filtered to remove the seasonal cycles. Contour interval is $0.05^{\circ} \mathrm{C}$, positive anomalies are shaded. While for the short period the response is somewhat similar between advective and nonadvective ocean, the response for the long period shows no resemblance at all.

agonal band originating in the first half of the pathway remains, while the amplitudes at the end of the pathway are reduced, suggesting that destructive interference took place. The 64-yr period in Fig. $5 \mathrm{~m}$ shows only very short phase lags between the two halves of the pathway. This suggests that for the longer NAO periods the fast advection dominates the temperature anomalies of the second half of the pathway.

To make the differences between advective and nonadvective ocean more obvious, we combined the heat content anomalies along the pathway for the dynamic (NAO-D) and the mixed layer (NAO-M) ocean in Fig. 6. Shown are the 4-yr, 16-yr, and 64-yr forcing periods. As can be seen from this diagram there is, for the short forcing period, some resemblance between the advective and nonadvective ocean in the form of a distorted checkerboard pattern. For the 16-yr forcing period we find that the advective ocean creates a strong impression of a propagating signal while the nonadvective ocean again responds with a checkerboard pattern. If the forcing 


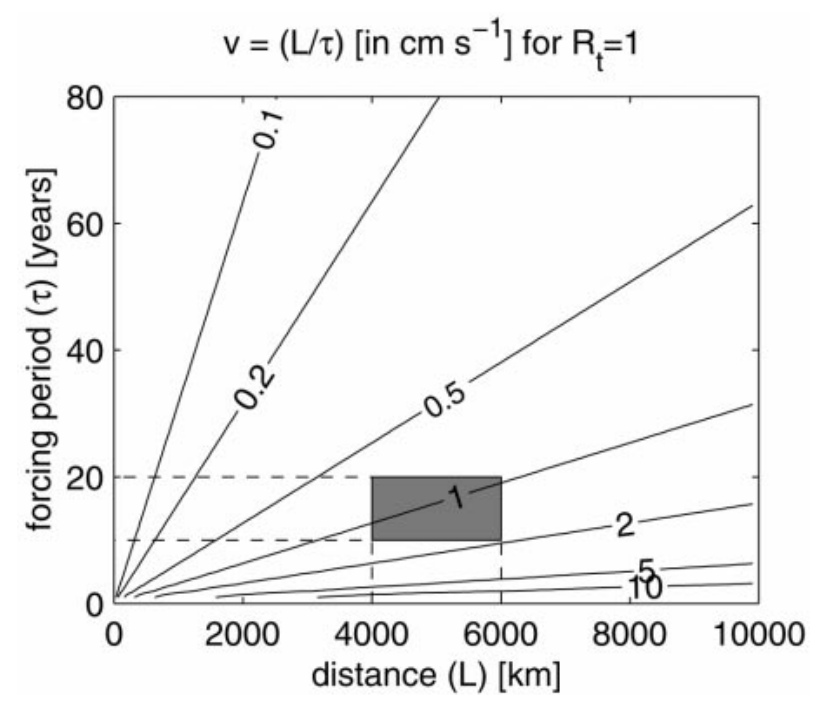

FIG. 7. This diagram shows the propagation speed that is necessary to obtain maximal response for any given length- and timescale of the forcing. The realistic range for the NAC marked by the shaded area.

anomalies are applied with a period of 64 years the responses of the advective and nonadvective oceans are very different. In the case of the advective ocean the heat content along most of the pathway varies in phase with the forcing while for the nonadvective ocean only the first half reacts in phase with the forcing while the second half is out of phase.

From Eq. (1) we can infer at which advection speed we can expect maximal response for any given spatial $(L)$ and temporal $(\tau)$ scale of the forcing. This is shown in Fig. 7. There the velocities necessary to obtain $R_{t}=$ 1 are contoured as a function of $L$ and $\tau$. This diagram can be used to obtain the forcing periods at which maximal response is to be expected for any given lengthscale of the forcing and propagation speed of the ocean. For the NAC with a propagation speed of about $1-2 \mathrm{~cm} \mathrm{~s}^{-1}$ and a lengthscale of 4000-6000 km maximal response is thus to be expected at about decadal timescales (see shaded area in Fig. 7).

In order to quantify the propagation speeds in our experiments we use simple harmonics to determine the phase lags of the temperature anomalies along the NAC pathway relative to the NAO forcing. We applied a fit of the interannual forcing harmonic to the temperature anomalies at each grid point of the pathway and then combined the phase and time lags in phase-distance and time-distance diagrams, respectively. This method is insensitive to the seasonal variability that we found in the previous analysis and is thus closer to an analysis of wintertime data, as used in most observational studies (Kushnir 1994; Hansen and Bezdek 1996; Sutton and Allen 1997).

Figure 8 includes the results for the forcing periods from 4 to 64 years and allows us to determine propa- gation speeds of the temperature anomalies for the different runs. The 2-yr period has been omitted because its phases are not well determined. The propagation speeds are then calculated by applying linear fits to the part of the pathway between 1200 and $6300 \mathrm{~km}$. The resulting velocities both in time-distance and phasedistance space are listed in Table 2 . They vary significantly with the forcing period. We find that for longer periods the speed in time-distance space decreases while it increases in phase-distance space. This is suggestive of the actual propagation being determined partially by advective processes and by external spatial variations in the forcing.

A thought experiment helps to understand how purely advective or purely externally forced regimes would manifest in the time-distance and phase-distance diagrams. Temperature anomalies are predominantly determined by the external forcing when $R>1$. If in this case the same spatial forcing pattern is applied with different forcing periods, lines with constant phase of the temperature anomalies like those in Fig. 8 would line up in a phase-distance diagram. In contrast the same constant phase lines would fan up in a time-distance diagram for the same set of experiments. If a similar set of forcings is applied to an advectively dominated ocean $(R<1)$ we would find that the constant phase lines line up in the time-distance diagram whereas they would fan in the phase-distance diagram.

In our set of experiments (NAO-D2 to NAO-D64) we have found that the propagation speeds of both surface (not shown) and upper 440-m temperature anomalies depend strongly on the period with which the sinusoidal forcing is applied. For the shorter periods of 4 to 8 years the phases of the heat content anomalies in Fig. 8a agree roughly with those of the mixed layer run. This means that for these short forcing periods the heat content anomalies are predominantly determined by the spatial variation of the forcing. For the very long period of 64 years the response is mostly in phase with forcing, except for the very last part of the pathway where the response occurs later but with only small amplitudes. The propagation speed for the long forcing periods is, however, slower than the pure advection speed, suggesting that the external forcing pattern still has some effect on the propagation. For intermediate forcing periods the response neither occurs in phase with the forcing nor does it show the pattern that is to be expected from the externally dominated mode. Instead, both external forcing and oceanic advection create a blended mode in which the apparent propagation speed is slower than oceanic advection but faster than the externally prescribed speed. While we have seen that over the whole pathway neither advection nor local forcing are solely responsible for the temperature anomalies, Fig. 8b reveals that in the central part of the pathway, that is, between the two NAD lobes, the temperature anomalies are dominated by advective effects. In this 

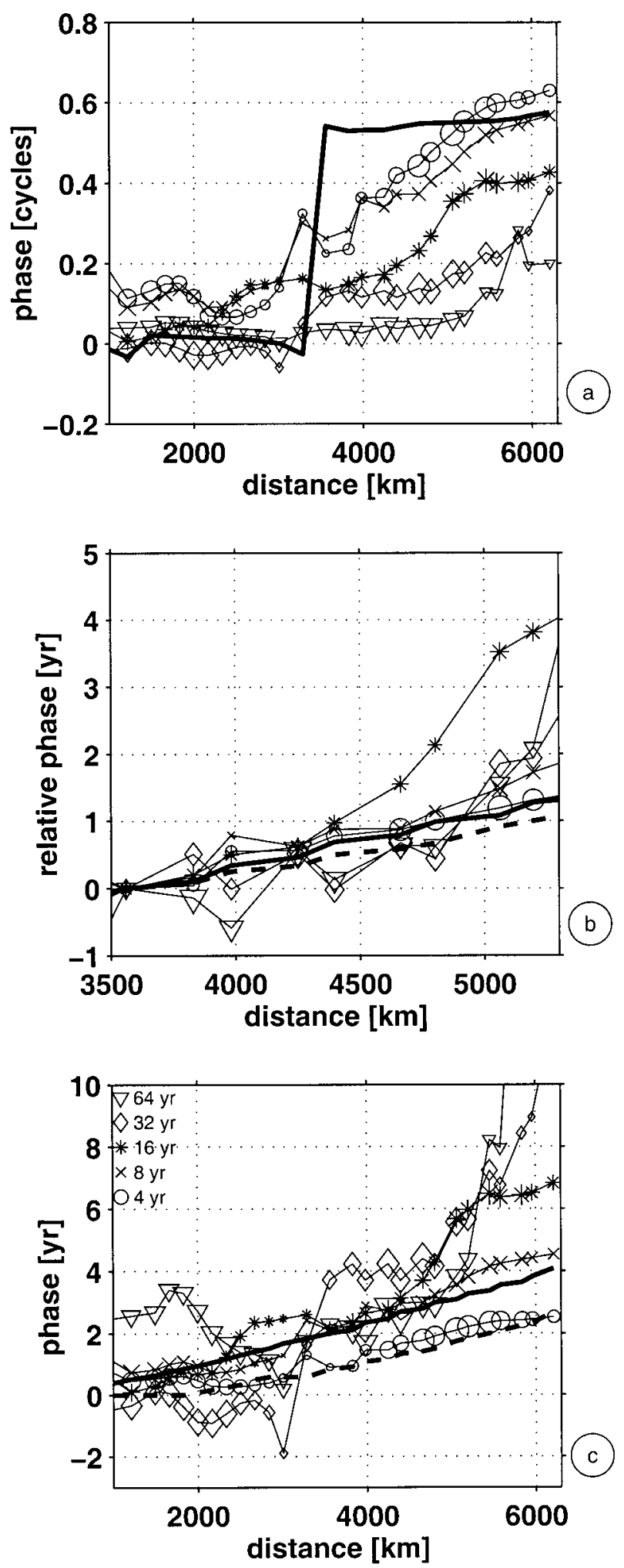

FIG. 8. Phases of the harmonic variation of the upper 440-m heat content relative to the forcing both in phase-distance and time-distance space. The phase-distance diagram (a) includes the phases of the heat content anomalies for the mixed layer model run with a forcing period of 16 years (solid line in a). The time-distance dia-
TABLE 2. Propagation speeds of the upper 440-m heat content anomalies for the different idealized NAO forcing periods. The speeds were calculated by a linear fit to the values in Figs. $8 \mathrm{a}$ and $8 \mathrm{~b}$ (restricted to the NAC pathway interval from 1200 to $5500 \mathrm{~km}$ ). Propagation speeds in time-distance space are decreasing with increasing length of the forcing period while the speeds in phase-distance space are increasing with the forcing period.

\begin{tabular}{lcc}
\hline \hline & \multicolumn{2}{c}{ Propagation speed } \\
\cline { 2 - 3 } Forcing period $(\mathrm{yr})$ & $\left(\mathrm{cm} \mathrm{s}^{-1}\right)$ & $\left(10^{6} \mathrm{~m} /\right.$ cycle $)$ \\
\hline 2 & 4.6 & 2.9 \\
4 & 5.6 & 7.0 \\
8 & 3.4 & 8.7 \\
12 & 2.5 & 9.4 \\
16 & 2.1 & 10.7 \\
24 & 1.9 & 14.3 \\
32 & 1.2 & 12.3 \\
64 & 0.8 & 15.6 \\
Advection & 5.4 & \\
TRACER & 6.1 & \\
Observations & 1.7 & \\
\hline
\end{tabular}

part of the pathway $(3500-4500 \mathrm{~km})$ the harmonic phases line up in time-distance space.

The propagation speeds determined by the linear fits enclose the speed calculated from observations (Sutton and Allen 1997). Best agreement between model and observations is found for a forcing period between 8 and 16 years. This frequency band coincides with the band for which we find maximal response in the model's subpolar gyre (Visbeck et al. 1998).

\section{c. Propagation along the NAC during the recent decades}

One question that arises after finding that NAO-like wind anomalies are able to create propagating temperature anomalies is how much of the anomalies found in the observations can be explained by the idealized NAO-like wind anomalies. We addressed this by conducting three additional experiments: The first experiment (NAO-HURR) is similar to the NAO-D series but now uses the observed NAO-index from Hurrell and van Loon (1997) instead of the idealized sinusoidal modulations. For the second experiment (NCEP) we added the full NCEP-NCAR reanalysis wind anomalies to investigate whether the inclusion of other, non-NAO related, variations of the winds improves the agreement between modeled and observed temperature anomalies. And in a third experiment (NCEP-M) we again used the full NCEP wind anomalies but applied them to the mixed layer only setup.

The Hovmöller diagram in Fig. 9a shows the winterly

grams (b and c) also include the graphs for the spreading of the maximum tracer concentration of experiment TRACER (dashed) and the propagation time calculated directly from the model's currents (solid). The time-distance diagram (b) displays a part of full pathway (c) in which advective spreading of the anomalies is dominant. 

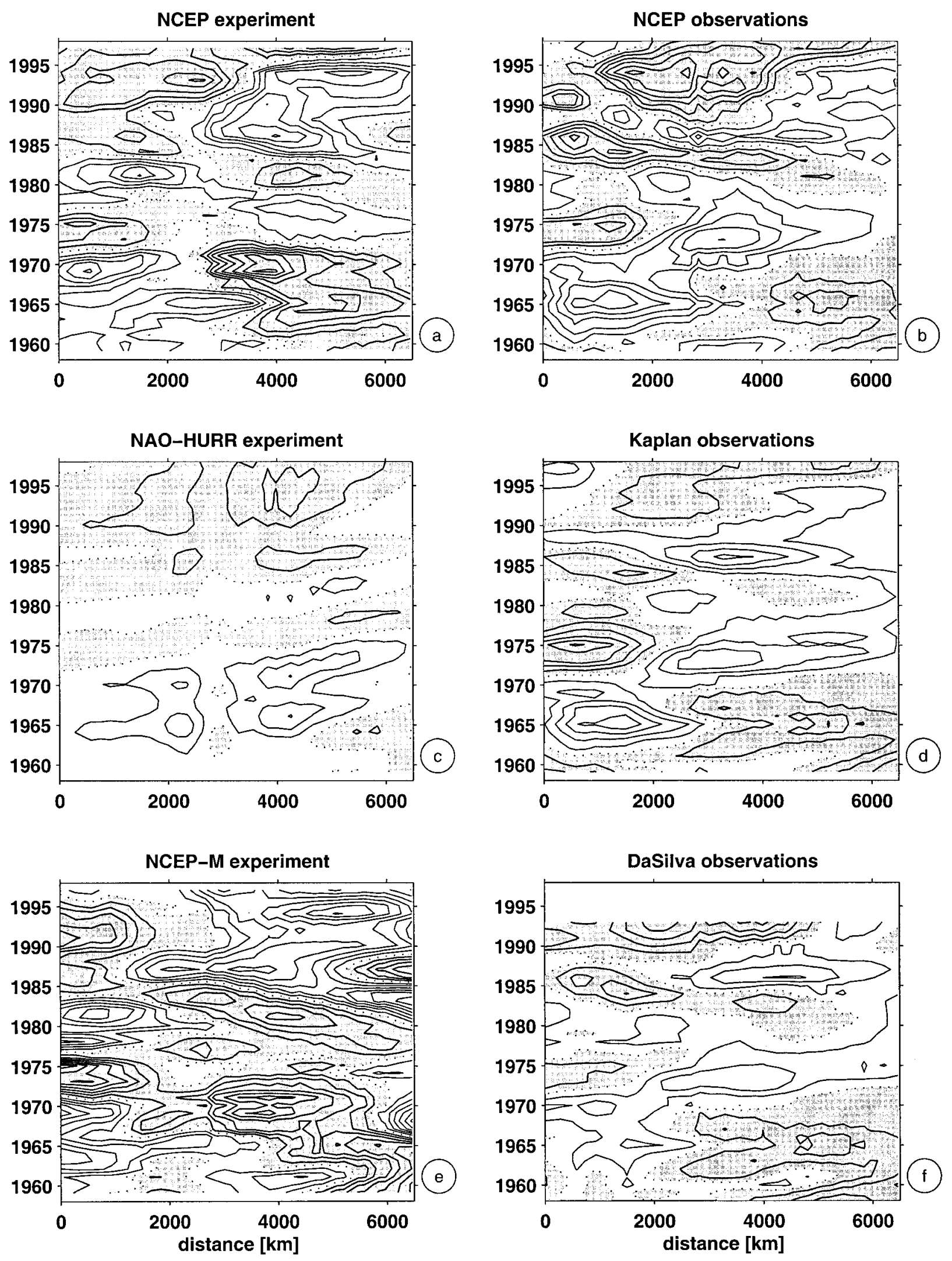
TABLE 3. Correlation between the various temperature anomalies shown in Fig. 9. High correlation levels ( $>0.76)$ are found between all observational datasets. Exp NCEP forced with the full NCEP wind and wind stress anomalies also yields relatively high correlations with the observations $(>0.50)$. The correlations between exp NAO-HURR and the observations are significantly smaller $(0.11-0.36)$, while the correlations for the not advective ocean experiment (NCEP-M) with the observations ranges typically between the full NCEP experiment and the NAO-HURR run $(0.32$ to 0.42$)$.

\begin{tabular}{|c|c|c|c|c|c|c|}
\hline & $\begin{array}{l}\text { NCEP } \\
\text { model }\end{array}$ & $\begin{array}{l}\text { NAO-HURR } \\
\text { model }\end{array}$ & $\begin{array}{c}\text { NCEP-M } \\
\text { model }\end{array}$ & $\begin{array}{c}\text { NCEP } \\
\text { obs }\end{array}$ & $\begin{array}{c}\text { Kaplan } \\
\text { obs }\end{array}$ & $\begin{array}{c}\text { DaSilva } \\
\text { obs }\end{array}$ \\
\hline NCEP & 1 & -0.11 & 0.75 & 0.50 & 0.56 & 0.56 \\
\hline NAO-HURR & & 1 & -0.14 & 0.36 & 0.11 & 0.27 \\
\hline NCEP-M & & & 1 & 0.32 & 0.42 & 0.35 \\
\hline NCEP & & & & 1 & 0.76 & 0.83 \\
\hline Kaplan & & & & & 1 & 0.77 \\
\hline DaSilva & & & & & & 1 \\
\hline
\end{tabular}

SST anomalies (Nov-Apr) of the experiment NCEP. In Figs. 9c and 9e the anomalies from experiments NAOHURR and NCEP-M are shown, respectively. Three different observational datasets covering roughly the same period of time are displayed in Figs. 9b,d,f. As before all diagnostics are performed along the NAC pathway (Fig. 3). Table 3 lists the correlation coefficients between the different datasets.

In all graphs in Fig. 9 we find significant variability on the decadal to interdecadal timescale. The observational datasets agree for most parts but differ in details (see Table 3). The observed temperature anomalies from da Silva et al. (1994), Kaplan et al. (1998), and NCEPNCAR indicate that cold temperatures occured between 1960 and 1970 along the North American east coast and that anomalies of similar amplitude were found some ten years later in the second half of the pathway. The propagation of anomalies between the first and the second halves of the pathway is, however, not as obvious from these graphs as in Sutton and Allen (1997). This is mainly caused by the different periods covered by our analysis (1958-98) and by that of Sutton and Allen (1997) (1946-88). In contrast to the findings by Sutton and Allen (1997) the anomalies during the 1980s observed by the NCEP-NCAR reanalysis and da Silva et al. (1994) seem to suggest a westward or upstream propagation. This prominent feature in our graphs is less pronounced in Sutton and Allen (1997), whose analysis also covers a strong downstream propagating anomaly in the 1950s.

The experiment NAO-HURR (Fig. 9c) is able to create decadal SST anomalies comparable to those found in observations. The temperature anomalies show a prominent propagation along the pathway of the NAC. Anomalies formed in the beginning of the pathway travel in about 5 years to the end of the pathway. The respective speed of about $4 \mathrm{~cm} \mathrm{~s}^{-1}$ is on the high end when compared to the results of the experiments
NAO-D (see Fig. 8 and Table 2). The anomalies, however, do not fully agree with the variations found in the observations. Good agreement is only found in the first half of the pathway, indicating that the NAO has a significant influence in this area. In the second half of the pathway the temperature anomalies created in the experiment NAO-HURR disagree with those found in the observations. This suggests that only a part of the observed temperature anomalies are due to the forcing variations associated with the NAO. In the next experiment we used the full variations of wind speed/wind vector and wind stress from the NCEP reanalysis. The temperature anomalies along the pathway obtained from this experiment are shown in Fig. 9a. They agree in contrast to the anomalies obtained in experiment NAOHURR rather well with the observed temperature anomalies. This supports the hypothesis that the full forcing anomaly fields are necessary to reproduce the observed fields.

In the experiment NCEP no propagation of temperature anomalies is visible. Figure 9a reveals, however, a clear out of phase relationship between the temperatures in the first and second half of the NAC pathway. A second look into the observed datasets shows that they too have a strong out of phase relationship between the anomalies in the two halves of the pathway. Their anomalies do, however, tend to be connected, which is responsible for the impression of propagation. Our experiment NCEP does not show this connection. The pattern of opposite temperature anomalies in the two halves of the pathway agrees very much with the pattern we have obtained for the idealized experiments with short forcing periods $(R>1)$. This is true even though the temperature variations in experiment NCEP occur predominantly on decadal and longer timescales for which we found propagating or even in phase response in the idealized experiments.

We thus find that the idealized NAO forcing anom-

$\leftarrow$

FIG. 9. Hovmöller diagrams of the winterly (NDJFMA) temperature anomalies along the NAC for the experiments NCEP (a), NAO-HURR (c), and NCEP-M (e) and for the observational datasets NCEP (b), Kaplan (d) and DaSilva (f). Contour interval is $0.2^{\circ}$, shaded areas denote positive anomalies. A 3-yr running mean has been applied to all datasets as has been done by Sutton and Allen (1997). 
alies (NAO-HURR) create strongly propagating temperature anomalies that, however, do not agree well with observations. Adding non-NAO anomalies (NCEP) creates on one hand a good agreement with observations, but causes on the other the disappearance of visible propagation. That oceanic advection still remains a necessary ingredient for the realistic reproduction of observed temperature anomalies can be seen in Fig. 9e and in Table 3. There it becomes apparent that the correlations of the temperatures of the mixed-layer only ocean model run (NCEP-M) with the observations reach only two thirds of those of the full experiment (NCEP). Nevertheless the simpler run (NCEP-M) does already reproduce several of the observed features. Overall, the results of these, more realistic, experiments are somewhat inconclusive.

\section{Conclusions}

We have performed various experiments with an ocean GCM of the North Atlantic coupled to an atmospheric mixed layer (Seager et al. 1995; Visbeck et al. 1998) in order to study the ocean's response to NAOlike wind anomalies. We found that the NAO-like forced runs are able to create temperature anomalies comparable to those found in observations (Visbeck et al. 1998). After their formation off the North American east coast the temperature anomalies propagate along the pathway of the NAC. Depending on the period with which the forcing was applied they cross the Atlantic in 5 to 10 years. This agrees well with the observations from Sutton and Allen (1997), who find them to cross the Atlantic in about 8 years.

Two basically different mechanisms are proposed for the formation and propagation of the temperature anomalies along the pathway of the NAC: local formation of temperature anomalies by changes in surface fluxes or by changes in the oceanic heat transport divergence and the advection of existing anomalies by the mean flow. Our experiments indicate that simple advection of temperature anomalies, created off the American coast, is not able to fully explain the temperature anomalies along the pathway of the NAC. Instead we found that, for NAO forcing periods up to 16 years, the spatial pattern of the direct response to the forcing variations can be found in the temperature anomalies along the pathway of the NAC. For the NAO-like forcing anomalies this pattern is dipolar with in-phase (positive temperature anomalies during a positive NAO index) formation of temperature anomalies in the first half of the pathway and out-of-phase (negative temperature anomalies during a positive NAO index) formation in the second half of the pathway. We also found that about two-thirds of the heat content anomalies of the layer 0-440 $\mathrm{m}$ were formed by changes in the oceanic heat transport divergence and only one-third by surface heat fluxes. They do, however, agree roughly in their spatial distribution, making their effects difficult to distinguish.
The two mechanisms have two different timescales for the propagation of the temperature anomalies. The timescale $L / v$ of the advection of an existing anomaly is determined by the ocean's mean currents $v$, which we found to be largely independent of the NAO-like forcing variations, and by the length of the pathway $L$. The timescale of the local formation of temperature anomalies is in contrast variable and determined by the NAO forcing period $\tau$. The propagative timescale for our experiments is about 10 years for one pass along the whole pathway. We find that for NAO periods shorter than the advective timescale $(R>1)$ the temperature anomalies are arranged in a pattern that can be explained by the local formation only. For NAO periods comparable to the advective timescale $(R \sim 1)$ the oceanic advection transports the temperature anomaly created in the first half of the pathway to the second half of the pathway in exactly the time in which the external forcing has switched from one state to the other, thus reinforcing the locally created anomaly. For NAO periods being longer than the advective timescale $(R<1)$ the advected temperature anomalies created in the first half of the pathway reach the second half during a time when the locally created anomalies are of opposite sign. Thus the two mechanisms work against each other and lead to some cancellation. The degree of cancellation depends, however, on the length of the NAO period and on the strength of the local formation at different parts of the pathway.

This result is much in agreement with the simple onedimensional atmosphere-ocean model presented by Saravanan and McWilliams (1997). They found that a spatially coherent and temporally stochastic atmospheric forcing (sinusoidal along the spatial domain) can lead to enhanced response of the ocean at a certain frequency. The parameter in control of the reponse strength is the ratio of the advective and damping timescales. For weak damping the peak response occurs when the forcing frequency equals the advection speed in the ocean divided by the prescribed lengthscale of the forcing $v / L$. This is the case when $R=1$.

The propagation speeds of $0.8-5.6 \mathrm{~cm} \mathrm{~s}^{-1}$ found in the experiments cover the observed speed of $1.7 \mathrm{~cm} \mathrm{~s}^{-1}$ (Sutton and Allen 1997). Interestingly the speeds in the model depend on the period with which the idealized forcing was applied. The observed and modeled propagation speeds agree in the model run with the forcing period for which the model's temperature variations are largest, suggesting that the preference for the decadal timescale anomalies might be responsible for the observed propagation speed.

When forced with more realistic variations of wind and wind stress we obtain results that suggest only limited predictability of temperature anomalies downstream in the NAC. For the model run NAO-HURR, in which we modulated the NAO-related wind variations with the observed NAO index, we obtained nicely propagating temperature anomalies. However, downstream in the 
NAC they do not agree with those found in observations. If we use the full wind and wind stress anomalies of the NCEP-NCAR reanalysis (i.e., include the nonNAO-related anomalies), we obtain closer agreement with observations for the whole pathway. Both observations and the experiment forced with the full NCEP anomalies exhibit predominantly out of phase relationships between the two halves of the pathway. Though, in our idealized experiments, we found out of phase relationships only for short forcing periods, the observations and the experiment NCEP exhibit them even over the interdecadal timescale.

Acknowledgments. G. Krahmann and M. Visbeck acknowledge support from NOAA under Grant NA86GP0301. G. Krahmann also obtained funding for a one year postdoctoral fellowship at LODYC, Paris, from the EEC Millennia Project ENV4-CT95 0101. G. Reverdin received support from PNEDC. The views expressed herein are those of the authors and do not necessarily reflect the views of NOAA or any of its subagencies.

\section{REFERENCES}

da Silva, A. M., C. C. Young, and S. Levitus, 1994: Atlas of Surface Marine Data 1994. Vol. 1: Algorithms and Procedure, Tech. Rep. 6, NOAA, 83 pp.

Deser, C., and M. L. Blackmon, 1993: Surface climate variations over the North Atlantic Ocean during winter: 1900-1989. J. Climate, 6, 1743-1753.

Hansen, D. V., and H. F. Bezdek, 1996: On the nature of decadal anomalies in the North Atlantic sea surface temperature. J. Geophys. Res., 101, 8749-8758.

Hellerman, S., and M. Rosenstein, 1983: Normal monthly wind stress over the World Ocean with error estimates. J. Phys. Oceanogr., 13, 1093-1104.

Hurrell, J. W., 1995: Decadal trends in the North Atlantic Oscillation: Regional temperatures and precipitation. Science, 269, 676-679.

- and H. van Loon, 1997: Decadal variations in climate associated with the North Atlantic Oscillation. Climatic Change, 36, 301326.

Kaplan, R., M. Cane, Y. Kushnir, A. Clement, M. Blumenthal, and B. Rajagopalan, 1998: Analyses of global sea surface temperature 1856-1991. J. Geophys. Res., 103, 18 567-18 589.

Kushnir, Y., 1994: Interdecadal variations in North Atlantic sea surface temperature and associated atmospheric conditions. J. Climate, 7, 141-157.

Latif, M., 1998: Dynamics of interdecadal variability in coupled ocean-atmosphere models. J. Climate, 11, 602-624.

Robinson, W., 2000: Review of WETS-The Workshop on ExtraTropical SST anomalies. Bull. Amer. Meteor. Soc., 81, 567-577.

Saravanan, R., and J. McWilliams, 1997: Stochasticity and spatial resonance in interdecadal climate fluctuations. J. Climate, 10, 2299-2320.

Seager, R., M. Blumenthal, and Y. Kushnir, 1995: An advective atmospheric mixed layer model for ocean modeling purposes: Global simulation of surface heat fluxes. J. Climate, 8, 19511964.

— , Y. Kushnir, M. Visbeck, N. Naik, J. Miller, G. Krahmann, and H. Cullen, 2000: Causes of Atlantic Ocean climate variability between 1958 and 1998. J. Climate, 13, 2845-2862.

Sutton, R., and M. Allen, 1997: Decadal predictability of North Atlantic sea surface temperature and climate. Nature, 388, 563567.

van Loon, H., and J. C. Rogers, 1978: Seesaw in winter temperatures between Greenland and northern Europe. Part I: Mon. Wea. Rev., 106, 296-310.

Visbeck, M., H. Cullen, G. Krahmann, and N. Naik, 1998: An ocean model's response to North Atlantic Oscillation-like wind forcing. Geophys. Res. Lett., 25, 4521-4524.

Walker, G. T., 1924: Correlations in seasonal variations of weather. IV. Memoirs of India Meteorolgical Department Rep. 24, 275332.

and E. W. Bliss, 1924: World weather V. Mem. Roy. Meteor. Soc., 4, 53-84. 